\title{
Scaling and linear response in the GOY turbulence model
}

\author{
Leo Kadanoff ${ }^{a}$, Detlef Lohse ${ }^{a, b, 1}$, Norbert Schörghofer ${ }^{a, *}$ \\ a The James Franck Institute, University of Chicago, 5640 South Ellis Avenue, Chicago, IL 60637, USA \\ ${ }^{\mathrm{b}}$ Fachbereich Physik, Universität Marburg, Renthof 6, 35032 Marburg, Germany \\ Received 28 March 1996; revised 20 June 1996; accepted 20 June 1996 \\ Communicated by F.H. Busse
}

\begin{abstract}
The GOY model is a model for turbulence in which two conserved quantities cascade up and down a linear array of shells. When the viscosity parameter, $v$, is small the model has a qualitative behavior which is similar to the Kolmogorov theories of turbulence. Here a static solution to the model is examined, and a linear stability analysis is performed to obtain response eigenvalues and eigenfunctions. Both the static behavior and the linear response show an inertial range with a relatively simple scaling structure. Our main results are: (i) The response frequencies cover a wide range of scales, with ratios which can be understood in terms of the frequency scaling properties of the model. (ii) Even small viscosities play a crucial role in determining the model's eigenvalue spectrum. (iii) As a parameter within the model is varied, it shows a "phase transition" in which there is an abrupt change in many eigenvalues from stable to unstable values. (iv) The abrupt change is determined by the model's conservation laws and symmetries.

This work is thus intended to add to our knowledge of the linear response of a stiff dynamical system and at the same time to help illuminate scaling within a class of turbulence models.
\end{abstract}

PACS: 47.27.-1; 47.27.Jv; 24.27.Eq; 05.45.+b; 02.10

\section{Introduction}

In turbulent flow a hydrodynamic system couples together many different length scales and thus shows in a single process a huge range of relaxation rates. There are a variety of simplified models of turbulence which are also intended to show this wide range of frequency and wave number scales. One such model goes under the inelegant acronym of "GOY". The model couples together a large number of shells, each with its characteristic scale of wave vectors and relaxation times. Shells are spaced logarithmically in wave vector. The $n$th shell is characterized by a single complex velocity, $U_{n}$, which then depends upon time, $t$. The model is a linked set of ordinary differential equations for all these $U_{n}$ with equations picked to mimic those of real hydrodynamic flow.

\footnotetext{
* Corresponding author.

${ }^{1}$ E-mail; lohse@cs.uchicago.edu. 
We do not know whether the model has much to do with turbulence. But it certainly illustrates the behavior of a stiff system. (Stiff systems are ones in which numerical simulations are made difficult by the effects of a huge range of relaxation rates.) In this paper, we describe the time dependence of the model in the simplest possible situation, the linear response to disturbances around a static solution. The response is described as an eigenvalue problem, with the response matrix being a large matrix which inherits the conservation laws, the scaling properties, and the symmetry principles of the GOY model. We look for the eigenvalues and eigenstates of the matrix. As we find them we see that the linear response, in turn, shows a considerable richness including scaling behavior and the analog of a phase transition. Much of this behavior can be understood in terms of the several symmetries and conservation laws of the original model.

The richness of behavior in this linear response theory serves to remind us that there is one area of scaling or similarity theory which has not been fully explored, the determination of eigenfunctions for matrices which have an underlying scaling structure. We also do not understand very much about turbulence, or even about the flow of information up and down dynamical linear chains. This paper is about these three not-fully-understood areas of applied mathematical science.

To start, we show the most interesting results of our study. We plot in Fig. 1, the eigenvalues of the linear stability in a sort of polar diagram in which the polar coordinates, $\theta$ and $r$, respectively, are the eigenvalues' phase and are proportional to the logarithm of the magnitude of the eigenvalue. (See Eq. (24) for a precise definition.) The two different parts of the plot show the response to a purely real disturbance (in part 1a) and to a purely imaginary disturbance (in part $1 \mathrm{~b}$ ). This distinction is meaningful because both the basic equations and the static solution are purely real. For this figure we have picked a particularly small value of the viscosity parameter so as to arrive at a simple scaling behavior. Indeed the simple spacing of the points in Fig. 1(a) shows that a simple multiplicative law generates the higher-order eigenvalues from the lower-order ones. That is why the points fall on a straight line with regular spacings. There is a tremendous range of scaling of the eigenvalues, and it all looks provocatively simple. This paper is mostly aimed at producing a partial explanation of these figures.

The paper starts with the introduction of the GOY model, and treats scaling for the static solution in Section 2. In Section 3 we discuss the linear stability analysis in general and apply it to the consideration of the scaling of the simpler, real component of the response. Also a relation between eigenvalues and conservation laws is derived. Section 4 discusses the linear stability in the imaginary response sector and the phase transition and scalings seen there. Section 5 summarizes the results. Footnotes and appendices discuss the findings that are not necessary for the main line of thought.

\subsection{The model}

The basic structure of GOY originated from Gledzer [1] was motivated by the cascade structure of turbulent eddies and conservation laws. Later, Ohkitani and Yamada [2] generalized the model and carried out numerical studies that revealed chaotic behavior and a dynamic scaling of velocity fluctuations. These studies have been extended in [3-6]. A popular introduction can be found in [7].

The basic ingredient is the hierarchical structure: The $n$th shell is characterized by a wave vector of length

$$
k_{n}=k_{0} \lambda^{n}, \quad n=1,2, \ldots, N
$$

with $\lambda>1$ and by a complex velocity mode $U_{n}$. The Navier-Stokes dynamics is mimicked by the following set of ODEs:

$$
\frac{\mathrm{d}}{\mathrm{d} t} U_{n}=F_{n}-C_{n}\left(U^{*}\right)-D_{n}
$$



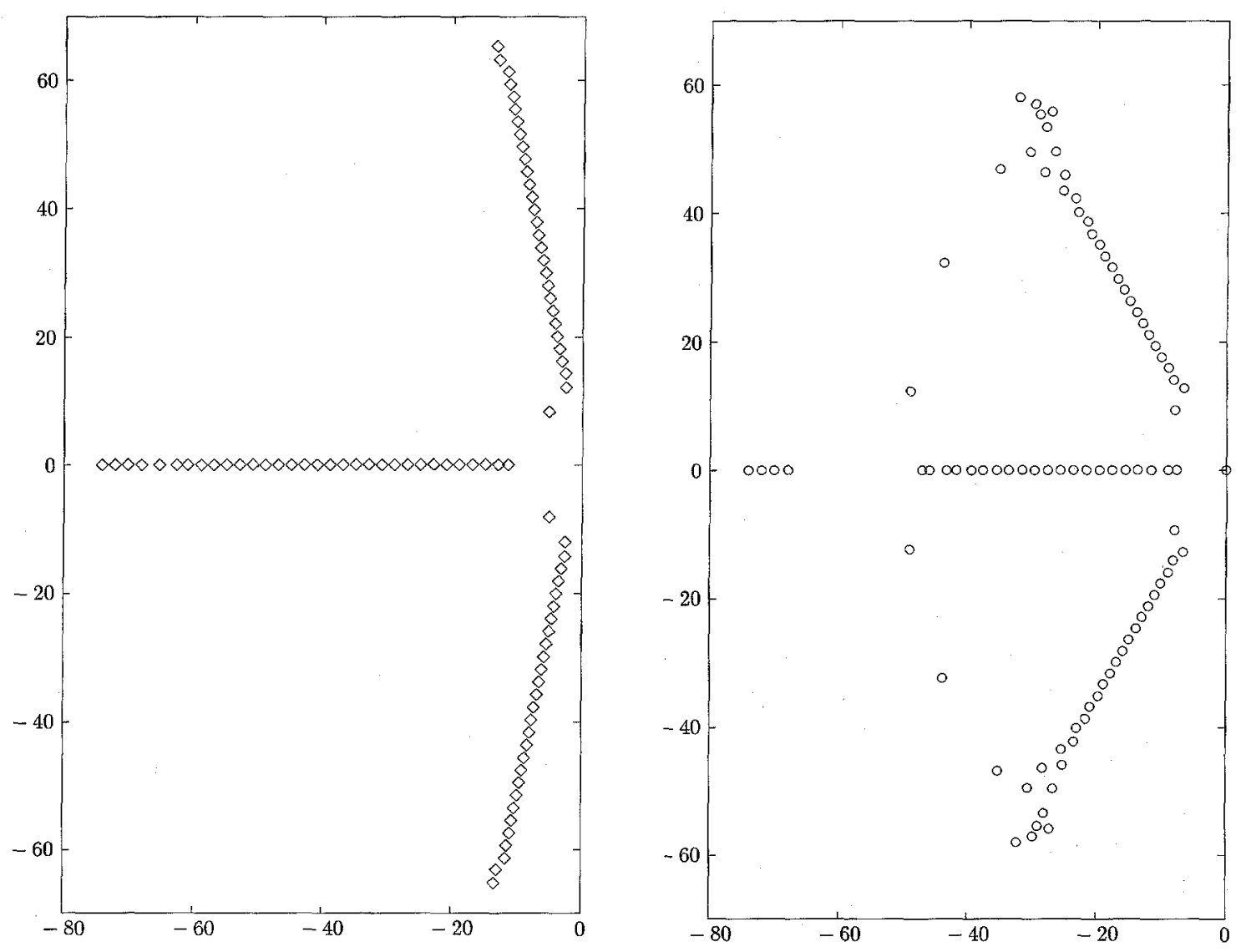

Fig. 1. Linear stability eigenvalues. The case considered is $\epsilon=0.3, v=10^{-3} 16^{-26}, N=90$. Here we plot the amplitude (diamonds) and phase (circles) of the eigenvalues in a kind of polar plot. The phase in this polar diagram is the phase of the eigenvalue. The radial coordinate is given by Eq. (24) which essentially produces the logarithm of the eigenvalue.

where the three terms represent, respectively, forcing, cascade processes and dissipation. (The $*$ indicates the complex conjugation.)

We pick a forcing on the first shell

$$
F_{n}=\delta_{n, 1} f
$$

Most previous studies [2,3,6,9] of the GOY model use a forcing on the fourth shell. Our choice of the first shell seems to give a simpler structure to the results. More details can be found in Appendix A. In our numerical work we shall choose $\lambda=2, k_{0}=\lambda^{-1}, f=1$ unless otherwise stated.

The dissipation term is

$$
-D_{n}=-\nu k_{n}^{2} U_{n}
$$

and is the $k$-space representation of the usual viscous dissipation process.

The cascade term couples the shell $n$ to its nearest and next nearest neighbors,

$$
C_{n}(U)=k_{n} U_{n+1} U_{n+2}-\epsilon k_{n-1} U_{n-1} U_{n+1}-(1-\epsilon) k_{n-2} U_{n-1} U_{n-2} .
$$




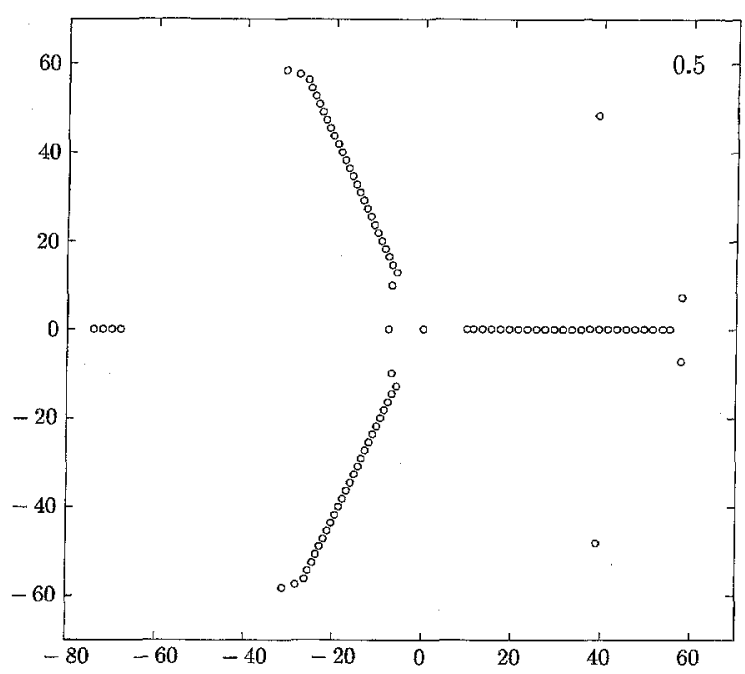

Fig. 2. The same as Fig. 1(b) except that now $\epsilon$ has the value 0.5 . (The amplitude matrix becomes eventually unstable only at $\epsilon \approx 0.558$. For small enough $\epsilon$ the moduli eigenvalues will not change much in the dynamics. These modes are unstable only for certain $v$ and only very slightly. For larger $\epsilon>0.56$ the moduli eigenvalue become increasingly unstable. We assume that the dimension of the chaotic attractor will increase correspondingly, but did not do a careful analysis on this.)

The boundary conditions are simply $U_{-1}=U_{0}=0$ and that the velocities go to zero as $n$ becomes large. (In our numerics we represent that by cutting off at some large shell numbered $N$ and then using the conditions $U_{N+1}=U_{N+2}=0$.) Since the static equations couple $n$ with the four immediately neighboring shells, we need four boundary conditions to define the problem. Two of the conditions are at the low- $n$ end, and two at the high. The nature of the boundary conditions will become crucial later on.

The model parameter $\epsilon$ determines the ratio between upscale and downscale coupling. It gives us a convenient tool for varying the model and seeing qualitatively different ranges of behavior.

In the inviscid, unforced limit there are two conserved quantities: the energy

$$
E=\frac{1}{2} \sum_{n}\left|U_{n}\right|^{2}
$$

and a second conserved quantity of the form

$$
H=\sum_{n} \frac{\left|U_{n}\right|^{2}}{(\epsilon-1)^{n}}
$$

that can be roughly associated with the helicity in fluid motion [6]. Even though the association is not perfect, we shall call this quantity the helicity in this paper (for a GOY type model with an improved representation of the helicity, see [8]).

The GOY model shows dynamical as well as static behavior. There is a static solution [9] of the GOY model ( $\mathrm{d} U / \mathrm{d} t=0$ ) in which the phases are zero. When we wish to point particularly to the static solution, we shall write it as $u_{n}$, while we use $U$ to refer to either the static or the dynamic case. In this paper, we study the static solution, $u_{n}$, and its linear stability properties.

The phases are not uniquely fixed for $u_{n}$ [9] which is part of a one parameter family of solutions. (We pick the particular solution which has $u_{n}$ real and positive.) The invariance in the phase will be important in our linear 


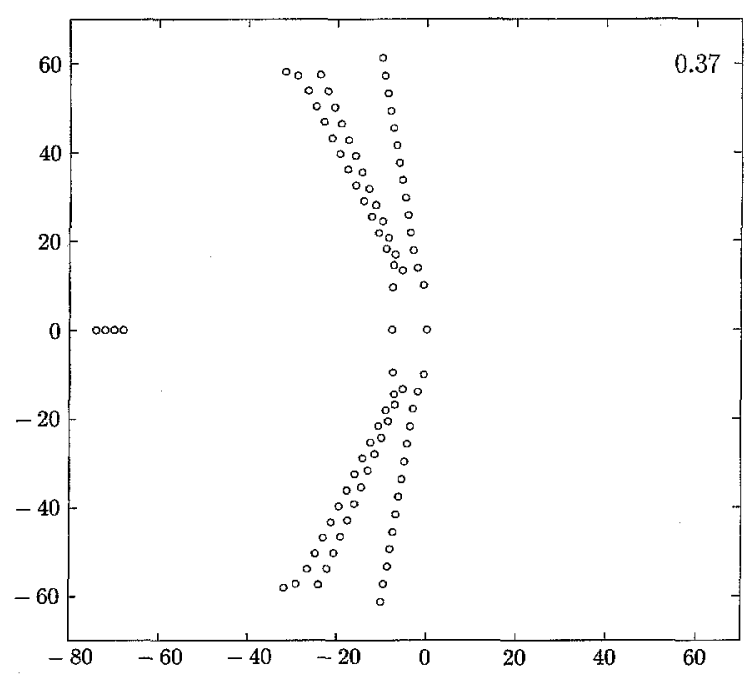

Fig. 3. The same as Fig. 1(b) except that now $\epsilon$ has its critical value, $\epsilon_{\text {bif }}$ which is about 0.37 . Notice that there are now six branches, all with complex eigenvalues. The equal spacing on each branch is evidence of scaling. But, evidently, the scaling is quite different from that shown in Figs. 1(b) and 2.

stability analysis. It gives rise to a zero eigenvalue. There are other approximate invariance properties which will be discussed when we get to the linear stability analysis.

\section{Scaling (of static solution)}

We are interested in understanding the behavior of the model in the limit as the viscosity becomes small. In that limit, there are three regions of $k$-space, or of $n$, called the stirring subrange (SSR), the inertial subrange (ISR), and the viscous subrange (VSR). The last is dominated by the viscosity term and has a velocity which decays very rapidly with $k$. (In the GOY model, the decay is exponential in a power [9] of $k_{n}$.) The SSR is naturally enough the range in which stirring is directly important. In our case this comprises only $n=1$. The ISR is the intermediate range of wave vectors between these two. Here, the behavior is dominated by the cascade term. In the center of the ISR, the solution is best described by using the product of three velocities:

$$
\Delta_{n}(u)=k_{n} U_{n} U_{n+1} U_{n+2} .
$$

In the region in which only the cascade term matters, this product is

$$
\Delta_{n}=A+B(\epsilon-1)^{n},
$$

where $A$ and $B$ are adjustable constants of integration, representing, respectively, the energy and helicity flux through the inertial range. We shall work with $\epsilon$ in the unit interval so that, for large $n$, the $A$ term dominates. In this region: ${ }^{2}$

$$
U_{n}=W_{n} k_{n}^{-1 / 3}
$$

\footnotetext{
${ }^{2}$ At present it is hardly possible to analyze the structure of the second, "fluxless" solution with $A=0$. It is unstable, so it is hard to obtain from the dynamical numerics. All solutions we have we essentially obtain from the dynamics which converged to a static solution (flux-solution K41). Then we continuously varied parameters and thus could also analyze the unstable solution. But they were continuously connected to stable ones.
} 

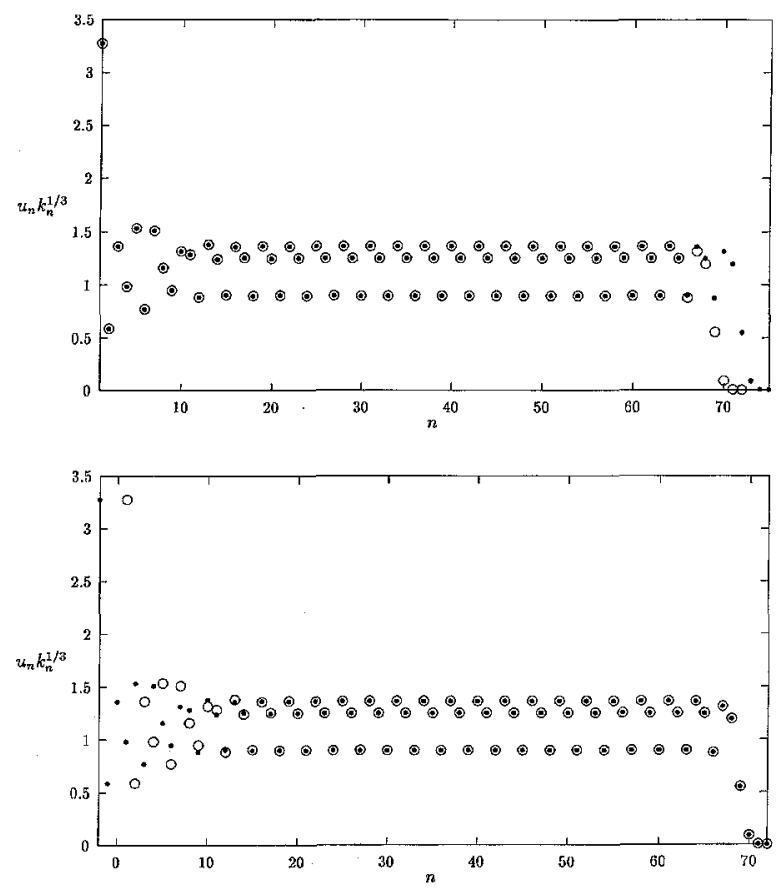

Fig. 4. We plot $W_{n}=k_{n}^{1 / 3} U_{n}$ against $n$. Notice how $W_{n}$ oscillates in the inertial range and how it falls off quite rapidly in the dissipative range. (The latter is for $n>N_{\mathrm{D}} \approx 67$.) The actual calculation is done for $\epsilon=0.3, v=10^{-3} 16^{-20}$ (circles) and $\nu=10^{-3} 16^{-21}$ (dots). The behavior of $W$ in these two simulations is compared. In the second (dots), $N$ is increased by three and $v$ is decreased by a factor of $\lambda^{-4}$. For all $n$ smaller than 56 or so the two calculations agree. (b) A comparison like that in (a) except that now $W_{n}$ is compared with $W_{n-3}^{\prime}$. These agree top plotting accuracy for all $n$ bigger than 20 or thereabouts.

where $W_{n}$ is a periodic function with period three. This behavior continues as $n$ increases until one enters the dissipative range, and $U_{n}$ begins to fall off very rapidly.

Notice that we have, as expected, four undetermined parameters in the solution. In the ISR these parameters are $A$ and $B$ and the two parameters which define the period-three oscillations.

The scaling behavior is illustrated in Fig. 4 which plots $W_{n}=k_{n}^{1 / 3} U_{n}$ against $n$ for a case in which $v$ has the value $10^{-3} 16^{-20}$. Notice how $U_{n}$ shows a simple scaling for large $n$ up to $n$ of about 67 , and then it nosedives. The nosedive occurs as $n$ increases above a dissipative threshold, which is achieved when the dissipation term and the cascade terms are roughly of equal size. Usually, $W_{n}$ is of the order unity in the inertial range so that this dissipative cutoff can be computed as a function of a threshold value of wave vector, called $k_{\mathrm{D}}$, which obeys

$$
k_{\mathrm{D}}^{-4 / 3} \sim v .
$$

The condition is thus that the viscous effects should dominate for values of $n$ larger than this dissipative threshold, that is,

$$
n>N_{\mathrm{D}} \sim-\frac{3}{4} \log _{\lambda} \nu
$$

There is a simple scaling theory which we can apply to this case. Since the static solution has an asymptotic period three [6], the system should be almost unchanged by the transformation: ${ }^{3}$

\footnotetext{
${ }^{3}$ The change in $N$ would be unimportant were we really working with very large values of $N$. For numerical convenience we work with $N$ only seven or eight larger than $N_{\mathrm{D}}$. This change in $N$ in Eq. (12b) eliminates effects produced by changing the cutoff. Throughout this
} 


$$
\begin{aligned}
& v \rightarrow v^{\prime}=v / \lambda^{4}, \\
& N \rightarrow N^{\prime}=N+3 .
\end{aligned}
$$

In particular, as one goes from the primed to the unprimed situation, the static solution should be basically the same at both ends, with the large, $n$ end being only modified by having smaller values of $u$. Let $u^{\prime}$ be the solution for the velocity in the situation changed according to Eqs. (12a) and (12b). The two scaling symmetries can be written as

$$
\begin{array}{ll}
\text { (S) } u_{n}^{\prime}=u_{n}\left(1+\mathrm{O}\left(k_{n} / k_{N_{\mathrm{D}}}\right)^{2 / 3}\right) & \text { for } N_{\mathrm{D}}-n \gg 1, \\
\text { (L) } \lambda u_{n+3}^{\prime}=u_{n}\left(1+\mathrm{O}(\epsilon-1)^{n}\right) \text { for } n \gg 1 .
\end{array}
$$

In these equations "S" stands for small $k$ and "L" refers to large $k$. Note that the first of these equations remains valid in the SSR while the latter remains valid in the VSR.

In Eqs. (13a) and (13b) we have included estimates of the error resulting from the terms we have not taken into account in constructing the scaling. In the small $k$-range, we do not include viscous effects, so the error estimate is the relative size of the viscous term. For large $k$, we do not include the helicity flux represented by the $B$ in Eq. (8), so this effect is put into the error. The global error is set by the sizes of each of these errors at the far ends of the VSR. One such error is the value of $v$. The other, and usually larger effect, is the effect of the helicity flux term, $B$, at the large- $n$ end of the ISR. This term has the order of magnitude,

$$
\text { error }=\mathrm{O}(1-\epsilon)^{N_{\mathrm{D}}} \text {. }
$$

This scaling error must be at least as large as the maximum of this error and $\nu$.

In terms of $W$, defined by Eq. (9), the two scaling equations imply, respectively, that $W_{n}^{\prime}=W_{n}$ and $W_{n}^{\prime}=W_{n+3}$. In the center of the ISR, both these scaling symmetries are valid. Then $W$ has a period-three symmetry:

$$
\text { (I) } W_{n}^{\prime}=W_{n}=W_{n+3} \text { for } n \gg 1 \text { and } N_{\mathrm{D}}-n \gg 1 \text {. }
$$

"I" refers to an intermediate range which occurs in the middle of the ISR.

To check our thinking, we calculate the static solution of the GOY model. Deviations from scaling can be seen by looking at the behavior of

$$
\begin{aligned}
& \delta_{\mathrm{S}, n}=1-W_{n} / W_{n}^{\prime}, \\
& \delta_{\mathrm{L}, n}=\left|1-W_{n} / W_{n+3}^{\prime}\right| .
\end{aligned}
$$

Both quantities are plotted in Fig. 5. Also shown in this figure are theoretical lines which show the errors defined in Eqs. (13a), (13b) and (14). Theory and experiment show excellent agreement.

\section{Eigenvalue spectrum}

\subsection{Linear response}

The next stage is to do linear stability analysis. We consider small deviations about the static solution $u_{n}$ by writing

$$
U_{n}(t)=u_{n}\left(1+\delta \Phi_{n} \mathrm{e}^{\sigma t}\right) .
$$

paper we use a prime to denote quantities changed by the transformation of Eqs. (12a) and (12b). 


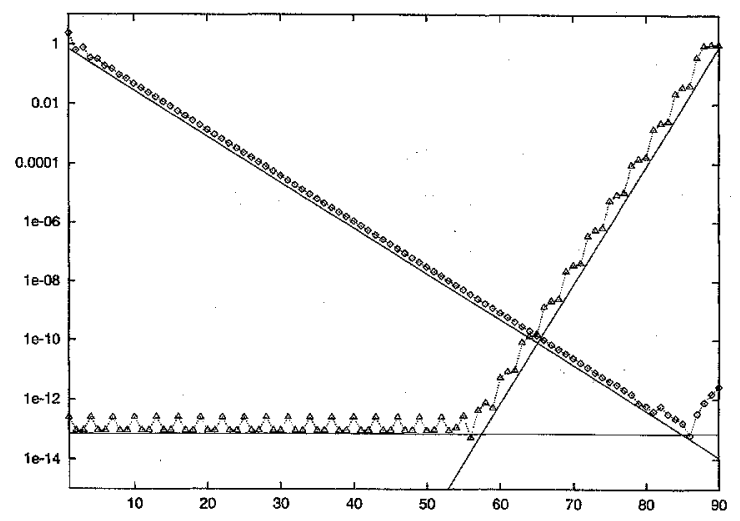

Fig. 5. The error in the scaling relations for the velocity. The deviation from unity of the ratio of two pieces of data in Eqs. (16a) (triangles) and (16b) (diamonds). The slope of the solid lines show the theoretical estimates of the error.

Since the static solution $u$ is real, the eigensolutions split neatly into oscillations of the phase and the amplitude, corresponding, respectively, to $\delta \Phi_{n}$ being real and imaginary. Then we have an eigenvalue equation

$$
\sigma \delta \Phi_{n}=\sum_{m} A_{n m} \delta \Phi_{m}
$$

We distinguish the two different cases with subscripts $M$ for magnitude and $\phi$ for phase. We use a superscript $j$ to denote which eigenvalue is being considered. In what follows we will always order the eigenvalues so that the magnitude of the eigenvalue increases with increasing values of the index $j$. Then the eigenstate will be very small for those shells which have $n$ considerably smaller than $j$. We will then argue that the behavior of the response matrix for $n$ and $m$ of order $j$ will play a large role in determining the eigenvalue.

Note that the response matrix $A$ has two parts. The dissipative part is

$$
D_{n m}=v \delta_{n m} k_{n}^{2}
$$

and the cascade response is

$$
C_{n m}=u_{m} \frac{\partial}{\partial u_{m}} C_{n}(u) .
$$

Now the structure of $A$ is different for the two kinds of response. For the magnitude response,

$$
A_{\mathrm{M}}=-C-D,
$$

while for the phase response

$$
A_{\phi}=C-D
$$

\subsection{Eigenvalue spectra}

At the center of the ISR, $u_{n}$ shows a simple scaling superposed on top of a period-three behavior. Thus, the response matrix also has a period-three scaling:

$$
C_{n+3, m+3}=\lambda^{2} C_{n, m} \text { for } n, m \text { in region I. }
$$


Consequently, if $C$ dominates the behavior of the matrices $A$ in the determination of eigenvalues in some range of $j$, then the eigenvalues would obey the scaling property

$$
\begin{array}{ll}
\sigma_{\mathrm{M}}^{j+3}=\lambda^{2} \sigma_{\mathrm{M}}^{j} & \text { for } j \text { in region } \mathrm{I}, \\
\sigma_{\phi}^{j+3}=\lambda^{2} \sigma_{\phi}^{j} & \text { for } j \text { in region } \mathrm{I} .
\end{array}
$$

In region $I$, then, the logarithms of the eigenvalues should be evenly spaced along straight lines with spacing of $\log \lambda^{2}$. Also, we might think that if $C$ dominates the behavior of the matrices $A$, then the phase and magnitude eigenvalues should be the same except for a minus sign. (See Eqs. (20a) and (20b)). The viscosity is unimportant in the entire region $\mathrm{S}$. Thus we expect

$$
\sigma_{\mathrm{M}}^{j}=-\sigma_{\phi}^{j} \quad \text { for } j \text { in region } \mathrm{S} .
$$

Fig. 1 shows the eigenvalue spectra for $\epsilon=0.3$ for both modulus and phase stability matrices $A_{\mathrm{M}}$ and $A_{\phi}$. As the distance between the eigenvalues $\sigma^{j}$ in the hierarchical GOY model grows roughly exponentially, it is hard to visualize the spectra in the complex plane. To have some kind of visualization we use a kind of polar representation in which the phase of the plotted point is exactly the phase of $\sigma$ while the distance from the center of the polar plot is given by

$$
r=\log _{\lambda}\left(1+2^{10}|\sigma|\right)
$$

The factor of $2^{10}$ is put in to enhance the visibility of eigenvalues with small values of $|\sigma|$. The plot has unstable eigenvalues showing up on the right-hand side of the origin while stable ones show up on the left. For large eigenvalues, even spacings on the plot mean that successive eigenvalues have ratios which are a constant. Thus even spacings are indicative of some kind of simple scaling.

In both the magnitude and the phase sector, the eigenvalues are arranged in several branches. Within each branch there are regions of even spacing, indicative of simple scaling. The magnitude eigenvalues seem particularly simple with three well-defined branches: A set of real eigenvalues and a pair of complex conjugate branches. All eigenvalues are stable. The phase eigenvalues show a more complex structure with what looks like more regions of simple scaling. Nonetheless both sets of eigenfunctions show the even spacing demanded by scaling. ${ }^{4}$ As one might expect, the minimal modulus eigenvalue is about the size of the entries in the first row of the cascade matrix $C$, the modulus of the second one is of the order of the second row, and so on. Generally, the modulus of the $n$th eigenvalue is about the size of the $n$th row of the matrix. This rule holds roughly until the eigenvalue with $j$ equal to $N_{\mathrm{D}}$ is reached whereupon the successive eigenvalues are real and have values of the order of the diagonal elements in the dissipation matrix, $D$. In the center of the inertial range the eigenvalues change from real to a complex conjugated pair and back to real and so on with a period three.

Thus, much of what we see is what we might expect. But not all Eq. (23) is completely inconsistent with the pictures we are seeing. This equation implies that if we have stable eigenvalues in the magnitude sector, we should have unstable ones in the phase sector. But all eigenvalues in Fig. 1 are stable. This result cannot be consistent with the notion that viscosity is unimportant for the eigenvalues in some region of the response. We have other difficulties. The pattern of phase response eigenvalues looks much more complex than the pattern of magnitude eigenvalues. Why should that be so?

\footnotetext{
${ }^{4}$ The existence of three branches is not due to the existence of a period three in the solution. One can assume an approximate solution $u_{n}=k_{n}^{-1 / 3}$, but the corresponding matrix still consists of three radial branches, although we have no period three in the "solution" any more.
} 
Another difficulty is associated with the prediction of Biferale $[11,12]$ that there would be a change in behavior at the special value of $\epsilon$ for which the contributions to the helicity sum in Eq. (6b) grow toward the high- $n$ end of the inertial range. The value is

$$
\epsilon_{\mathrm{bif}}:=1-\lambda^{-2 / 3} \text {. }
$$

We have just seen that the model is stable for $\epsilon=0.3$, which lies below the Biferale value $\epsilon$ approximately 0.37 . Look at Fig. 2, which is the analog of Fig. 1(b) but now for $\epsilon=0.5$. The magnitude spectrum looks much the same as before, but there is a qualitative change in the phase spectrum. Now, there are a large number of unstable eigenvalues in the phase spectrum. We interpret what we are seeing by saying that there is a qualitative change in properties of the asymptotic ( $v$ goes to zero) model at $\epsilon=\epsilon_{\mathrm{bif}}$. For $0<\epsilon<\epsilon_{\mathrm{bif}}$ there are at most a finite number of unstable eigenvalues. At $\epsilon$ just above $\epsilon_{\text {bif }}$ the system acquires an infinite number of unstable eigenvalues. Fig. 3 shows the behavior of the spectrum at the Bifarale point. Once again the phase spectrum has a qualitatively new character, while the magnitude spectrum remains much the same as before. We want to understand better how this behavior arises from the linear stability analysis.

\subsection{Eigenvalues in the magnitude sector}

The scaling behavior of the eigenvalues in the magnitude sector is given by the very same ideas which we already used in our analysis of $u_{n}$. As we shall see, some new ideas will be required for the phase sector. For this reason, we shall dispose of the magnitudes here and move on to a more extended discussion of phase eigenvalues in Section 4.

The general structure of the eigenstates is illustrated in Fig. 6. Here we look at right eigenvectors with $j=26$. The eigenvalue equation in the magnitude sector is

$$
\sigma^{j} \delta \Phi_{n}^{j}=-\sum_{m}\left(D_{n m}+C_{n m}\right) \delta \Phi_{m}^{j}
$$

For small $n$ the eigenvector is very small. In fact, the eigenvector must decrease rapidly as $n$ decreases to enable the eigenvalue term from swamping the right-hand side of the equation. This decrease is a falloff from a plateau which occurs at $n$ about equal to $j$. At this point, the eigenvalue term and the cascade term in Eq. (26) are about equal in size. As $n$ increases still further, the three parts of the cascade term each become much larger than the

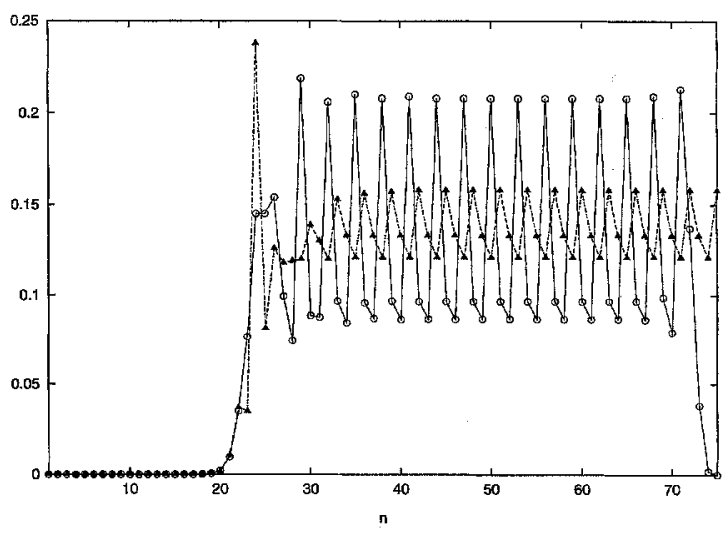

Fig. 6. The general structure of right eigenvectors for the the amplitude $\delta \Phi_{n}^{(26)} u_{n} k_{n}^{1 / 3}$ (solid) and phase $\delta \Phi_{n}^{(26)}$ (dashed line) $\left(N=75, \epsilon=0.3, v=10^{-3} 16^{-21}\right)$. In both cases the modulus of the eigenvector is displayed. The amplitude eigenvector gets damped when it enters the viscous range, while the period three of the phase eigenvector continues into the viscous range. 
eigenvalue term. To ensure the cancellation of the different parts of the cascade term the eigenvector settles down to an oscillation with period three, which holds throughout the entire ISR. Finally as $n$ enters the VSR, the eigenstate once again falls off quite rapidly. The behavior of the phase eigenstate is much the same, except that there is no falloff in the VSR.

One can describe the same process in physical terms by saying that the eigenvalue term adds energy to the system which then cascades toward the VSR, where the energy is dissipated.

Now one can see why the dissipation plays such a large role in determining the behavior of the eigenvalues. The deviation $\delta \Phi_{n}^{j}$ produces a change in the conserved quantities, both the helicity and the energy. This change cascades down through the ISR toward the VSR. Because of the conservation of energy this cascade is not damped and remains constant in size until the VSR is reached. Since the eigenvector remains constant into the VSR, the VSR behavior serves as a kind of large $n$ boundary condition on the eigenstate.

A simple count shows how this works. For large $n$ in the ISR, $\delta \Phi_{n}$ is periodic with period three. Then there are two parameters which describe the wave function:

$$
\begin{aligned}
& p_{1}^{j}(k)=\frac{\delta \Phi_{3 k+1}^{j}}{\delta \Phi_{3 k}^{j}} \\
& p_{2}^{j}(k)=\frac{\delta \Phi_{3 k+2}^{j}}{\delta \Phi_{3 k}^{j}}
\end{aligned}
$$

In our previous work [9] we found that there were two conditions upon the large- $n$ velocities, required to keep these velocities from blowing up deep into the VSR: Two parameters, two conditions. Everything is determined. Thus we might expect that all eigenstates with sufficiently small $j$ would have the very same values of the parameters for large enough $n$ in the ISR. Table 1 serves to check this point. In this table we have shown values of the ratio of these parameters for various different eigenstates. According to the theory, the ratio should be unity. Clearly the theory works well for the magnitude eigenstates. (Table 1 also shows that the constancy of the parameters does not work for the phase eigenstates, but that story will be told later.)

Table 1

Behavior of parameters for eigenstates

\begin{tabular}{lllllrr}
\hline$j$ & $n$ & \multicolumn{2}{l}{ Magnitude } & & Phase \\
\cline { 3 - 5 } \cline { 5 - 6 } & & $\operatorname{Re}\left(p_{1}^{j}(n) / p_{1}^{j+1}(n)\right)$ & $\operatorname{Re}\left(p_{2}^{j}(n) / p_{2}^{j+1}(n)\right)$ & & $\operatorname{Re}\left(p_{1}^{j}(n) / p_{1}^{j+1}(n)\right)$ & $\operatorname{Re}\left(p_{2}^{j}(n) / p_{2}^{j+1}(n)\right)$ \\
\hline 18 & 35 & 1.02897 & 0.99597 & 0.02867 & 0.81927 \\
18 & 45 & 1.00132 & 1.00041 & -4.83889 & 22.2840 \\
18 & 50 & 0.99986 & 1.00002 & 0.02867 & 0.81891 \\
18 & 55 & 1.00004 & 1.00002 & 0.31876 & -0.00242 \\
18 & 62 & 1.00000 & 1.00000 & 0.02867 & 0.81891 \\
18 & 70 & 1.00000 & 1.00000 & 0.31876 & -0.00242 \\
13 & 16 & 75.57590 & 2.08638 & -0.58804 & -51.81228 \\
13 & 35 & 0.99648 & 1.00048 & 40.53829 & -0.11043 \\
13 & 45 & 0.99984 & 0.99995 & -0.00677 & 0.02073 \\
13 & 55 & 1.00000 & 1.00000 & -0.42772 & -51.26321 \\
13 & 71 & 1.00000 & 1.00000 & 40.53836 & -0.11044 \\
\hline
\end{tabular}

Note. Here $j$ refers to the $j$ th eigenfunction while $n$ describes the components of that eigenfunction. These two parameters describe the large- $n$ behavior in the ISR. They are fixed by the value of $v=10^{-3} 16^{-21}$ in the magnitude sector but vary considerably in the phase sector. The ratios are essentially the same for the $n$ values in between the given values. (Correlations between phase values with $n$ different by a multiple of 3 stem from the period three in the eigenvectors.) 

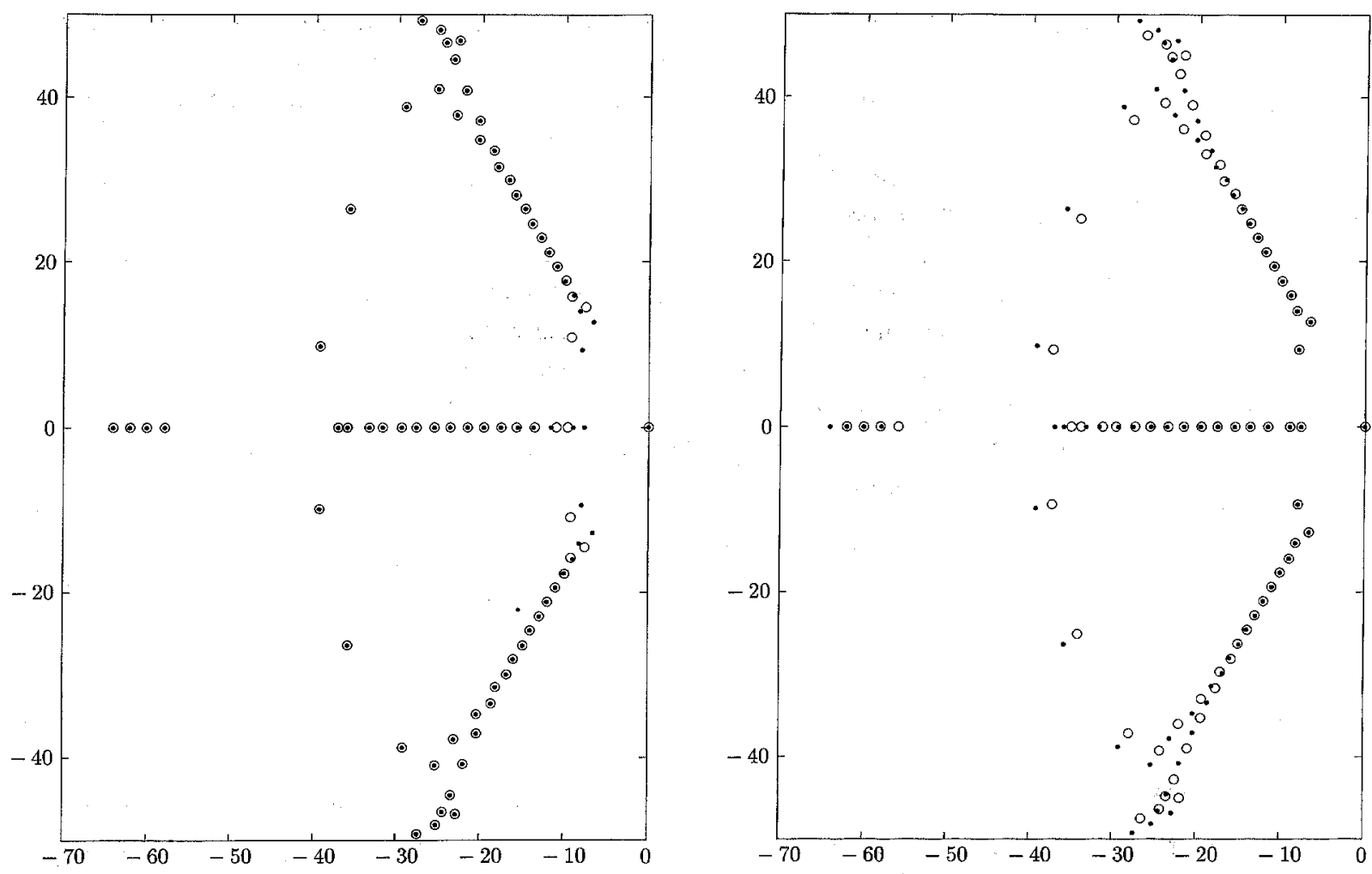

Fig. 7. (a) Eigenvalues of the phase matrix for $\epsilon=0.3, N=90$, and $N=93$. One can see three main branches and the validity of scaling law S. (b) The same data, but the eigenvalues for $N=90$ are multiplied by 4 . Scaling law $\mathrm{L}$ is at work.

Thus, the matching into the VSR uses two of the four boundary conditions on our linear chain problem. A very similar mechanism sets the other two boundary conditions in the regions in which $n$ is of order $j$. There are two quantities which can determine the behavior of the eigenfunction in this region. The first is the variation in helicity coefficient $B$ produced by the disturbance. This variation produces a term in $\delta \Phi_{n}^{j}$ which varies as $(\epsilon-1)^{n}$ and which then grows to be of relative order unity when $n$ is of order $j$. By setting this coefficient, and also the value of the $\sigma^{j}$, one has two coefficients at ones command. These two are just enough to ensure that the eigenfunction decays, rather than grows, as $n$ goes to one.

There is only one important difference between the problem of determining the static solution $u_{n}$ and the eigenfunctions. For the former, we have forcing on the first shell. In the latter, the important forcing is produced by the eigenvalue term, and occurs on the $j$ th shell. The scaling analysis for the $j$ th eigenvalue is modified because the range of the cascade becomes $j$ to $N_{\mathrm{D}}$ rather than the 1 to $N_{\mathrm{D}}$ that we used in the analysis of the velocity. Thus the scaling rule, with corrections, becomes on the small- $j$ end

$$
\left.\sigma^{\prime(j)}=\sigma^{(j)}\left(1+\mathrm{O}\left(k_{j} / k_{N_{\mathrm{D}}}\right)^{4 / 3}\right)\right) \text { for } j \text { in region } \mathrm{S} .
$$

Here, as before, the prime indicates a decreasing in the viscosity by a factor of $\lambda^{4}$ together with a shift in the cutoff, $N$. The corresponding result on the large $-j$ end is the statement

$$
\sigma^{(j+3)}=\lambda^{2} \sigma^{(j)}\left(1+\mathrm{O}(\epsilon-1)^{j}\right) \text { for } j \text { in region } \mathrm{L} .
$$




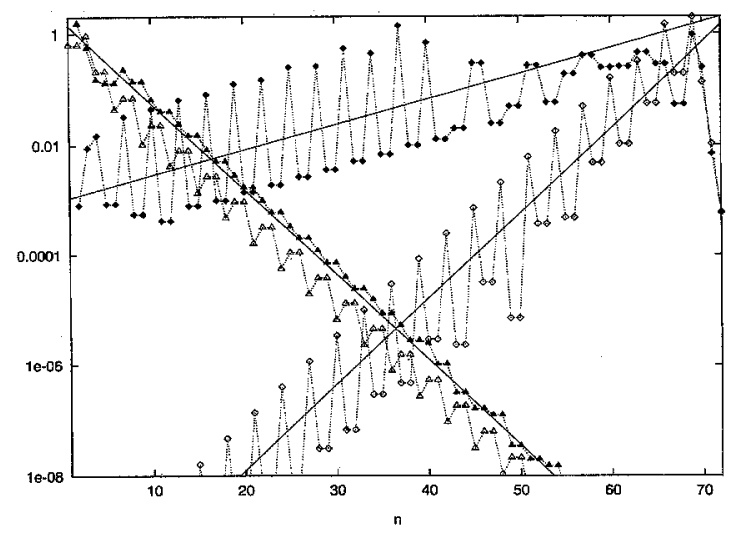

Fig. 8. Errors in the scaling laws for the eigenvalues, Eqs. (28a), (43a) and (28b), (43b); $\epsilon=0.3$. Here we plot $|R|-1$ where $R$ is the ratio of the two sides of the equation. The diamonds describe the ratio in Eqs. (28a) and (43a) while the triangles do the same for Eqs. (28b) and (43b). The unfilled symbols are for the amplitude matrix and the filled ones stand for the phase matrix. Lines are theoretical estimates of the errors. (Above $\epsilon_{\text {bif }}$ the errors undergo some modifications.)

Both together give the scaling law:

$$
\sigma^{\prime(j+3)}=\lambda^{2} \sigma^{(j)} \text { for } j \text { in region } \mathrm{I} \text {. }
$$

To check our analysis, we should check these rules. The first check, done in Fig. 7(a), is to plot overlays of the primed and unprimed eigenvalues on polar plots like that in Fig. 1. We show the phase eigenvalues here, but the scaling fits are even better for the magnitude eigenvalues. The two spectra overlay precisely at small $j$, as expected, and fit badly for large $j$. In the second check, one plots overlays of $\sigma$ and $\sigma^{\prime} / \lambda^{2}$ as shown in Fig. 7(b). This overlay shows agreement between the two for large eigenvalues but not for small. A more careful check is to take the ratio of the nearby eigenvalues in these two figures. Call this ratio $R$. The quantity $|R|-1$ is a quantitative measure of the errors in our statements (28a) and (28b). Fig. 8 plots this ratio versus $j$ and shows that the order errors vary as stated in these equations.

Thus, one can feel that the magnitude response is understood reasonably well.

\subsection{Eigenvalues and conservation laws}

One basic principle about this model is that the dissipation can play a large role in determining the ISR behavior. To see this fact in more detail, we examine the effect of the conservation laws for energy and helicity upon the eigenvalue analysis.

Let us recollect that the conservation laws for the system can be expressed as

$$
\sum_{n} U_{n} C_{n}(U) h^{n}=0
$$

Here $h$ is a quantity which defines the two conservations. For energy conservation $h=1$; for helicity

$$
h=(\epsilon-1)^{-1} .
$$

For the $j$ th right eigenvector $\delta \Phi^{j}$ and its eigenvalue $\sigma^{j}$, we have an eigenvalue equation of the form

$$
\sigma^{j} \delta \Phi_{n}^{j}=\sum_{m}\left( \pm C_{n m}-D_{n m}\right) \delta \Phi_{m}^{j}
$$


Here the minus sign corresponds to the magnitude eigenvalues and the plus to the phase eigenvalues. Multiply by $\left(u_{n}\right)^{2} h^{n}$ and sum over all $n$ to obtain

$$
\sigma^{j} \sum_{n}\left(u_{n}\right)^{2} h^{n} \delta \Phi_{n}^{j}= \pm \sum_{n m} h^{n}\left(u_{n}\right)^{2} C_{n m}(u) \delta \Phi_{m}^{j}-v \sum_{n} h^{n}\left(u_{n}\right)^{2} k_{n}^{2} \delta \Phi_{n}^{j} .
$$

The conservation identity (30) is true for any $U$ and hence also for $U=u(1+\delta \Phi)$. Since $\delta \Phi$ is a small perturbation we can expand around $u$.

$$
0=\sum_{n} h^{n} U_{n} C_{n}(U)=\sum_{n} h^{n}\left[u_{n} C_{n}(u)\left[1+\delta \Phi_{n}^{j}\right]+\sum_{n m}\left(u_{n}\right)^{2} h^{n} C_{n m}(u) \delta \Phi_{m}^{j}\right]
$$

In this way we have expressed the Jacobian (19b) in terms of the cascade itself. Eqs. (30), (34), and (2) yield

$$
-\sum_{n m} h^{n}\left(u_{n}\right)^{2} C_{n m} \delta \Phi_{m}^{j}=\sum_{n} h^{n} u_{n} C_{n} \delta \Phi_{n}^{j}=\sum_{n}\left(-D_{n}+F_{n}\right) u_{n} h^{n} \delta \Phi_{n}^{j}
$$

Now we can rearrange the eigenvalue equation. In the case of magnitude response the dissipation term in Eq. (35) adds to the dissipation term in Eq. (32) to give us an identity for the eigenvalue:

$$
\sigma_{\mathrm{M}}^{j} \sum_{n} h^{n}\left(u_{n}\right)^{2} \delta \Phi_{n}^{j}=-2 v \sum_{n} h^{n}\left(u_{n} k_{n}\right)^{2} \delta \Phi_{n}^{j}+f h u_{1} \delta \Phi_{1}^{j} .
$$

The left-hand side of this equation is the rate of decay of the conserved quantity, as determined by the eigenvalue. On the right-hand side we see that the decay is (naturally enough) not produced by the cascade but only by the dissipation through viscous damping and also by the addition through the external force, $f$. Thus we understand once more that the dissipation must have a crucial role to play.

If we go through the same calculation for the phase response, the result is totally different. Instead of adding to one another, the dissipation terms cancel out (!), leaving us with the identity

$$
\sigma_{\phi}^{j} \sum_{n} h^{n}\left(u_{n}\right)^{2} \delta \Phi_{n}^{j}=-f h u_{1} \delta \Phi_{1}^{j} .
$$

Compared to the magnitude sector, the phase sector shows a quite different form for the conservation law identities. We can no longer say that dissipation produces decay. Instead we say that the relevant quantity is added through the force term and then changes in time because of this addition. We now turn to a more detailed consideration of the phase sector.

\section{Phase response}

\subsection{Establishment of phase transitions}

From what we have seen, both $u_{n}$ and the entire magnitude sector of the linear response vary smoothly as $\epsilon$ passes through the Biferale value given by Eq. (25). When $\lambda=2$, this transitional value is 0.37 . The story is different for the phase response. Whenever $\epsilon$ passes through the critical value, then there is a quite apparent change in the structure of the eigenvalues. As the viscosity goes to zero, this change involves having a large number of eigenvalues pass from being stable to unstable. In the asymptotic limit, one third of the entire set of ISR eigenvalues undergo such a passage at this point.

To see the evidence for this proposition, return to Figs. 1(b), 2, and 3. These pictures, respectively, apply below, above, and at the phase transition in $\epsilon$. Away from the phase transition, the phase eigenvalues fall into two classes: 
The eigenvalues fall onto three lines of constant phase: one real and two with opposite phases. Others, the "deviating eigenvalues" do not seem to fall into the simple pattern, and have phases which change from eigenvalue to eigenvalue. Since we are interested in scaling properties, we want to know something about the pattern which arises when the viscosity is taken to zero. An examination of the spectrum shows that its three main branches grow longer as $v$ becomes smaller, but that the number of deviating eigenvalues does not grow. Fig. 9 presents a counting of eigenvalues in the phase response sector. They are divided into the following categories: real, "constant phase" complex, and deviating eigenvalues. They are then counted at different values of $N_{\mathrm{D}}$. We see that the number of deviating eigenvalues remains the same as we change the number of shells in the ISR. The same analysis - and result - applies above the transition. Consequently, for $v \rightarrow 0$ the number of deviant eigenvalues becomes negligible compared to the number in the three main branches. In the asymptotic limit, each of the main branches has one third of the total eigenvalues. Thus, the deviating eigenvalues are not a scaling limit phenomenon, but rather a transient which defines the approach to scaling at the ends of the ISR.

There is a main branch on the real axis both above and below the transition. Above the transition, this branch is unstable; below it is stable. At the critical point, we have a change involving, in the $v \rightarrow 0$ limit, an infinite number of eigenvalues.

Fig. 10, together with Figs. 1(b), 2, and 3, presents the flow of phase eigenvalues as $\epsilon$ goes from below to above the transition. We start at Fig. 10(a) with the familiar spectrum at $\epsilon=0.2$. Real eigenvalues collide and turn to complex ones and these (so created) deviating group of eigenvalues wanders towards the imaginary axis. If we had chosen a larger system, the number of deviating eigenvalues would still be the same, and only the straight branches would gain in members. The smaller the system the more is the discontinuous transition smeared out by finite size effects. (For finite $v$ also the critical point $\epsilon_{\mathrm{c}}$ weakly depends on $v$ and slightly deviates from $\epsilon_{\mathrm{bif}}$, see Fig. 11 . These finite size effects spoil the regularity of the phase transition. A related transition with less disturbing finite size effects is discussed in Appendix B.)

As the deviating group is close to the phase transition the two conjugate straight branches of eigenvalues split into two (Fig. 3), causing a breakdown of scaling law $S$. A scaling of the form $S^{2}$ (i.e., make use of the symmetry $n \rightarrow n+6$ ) however, is still valid. The scaling of the solution and of the magnitude eigenvalues does not break down at this point.

Above the transition Fig. 10(d) the deviating group has curved in the other direction and the eigenvalues return to the now unstable side of the real axis.

There is a hidden structure of the deviating group not easily seen in our representation (24); these eigenvalues fall on a straight line in a $\log (|\sigma|)-\arg (\sigma)$ plot. The inset in Fig. 9 shows (the upper half of) the deviating group and the real eigenvalues for $\epsilon=0.33,0.37$, and 0.4 . A dotted line marks the border of instability at $\frac{1}{2} \pi$. Remember again that only branches with constant phase (here horizontal) will contribute in the $N \rightarrow \infty$ limit. From Fig. 9 (inset) it is evident that with $N \rightarrow \infty$ instability will occur immediately above $\epsilon_{\text {bif }}$, since an arbitrary small tilting suffices to produce unstable real eigenvalues. Moreover, for $v \rightarrow 0$ there is not even a finite number of unstable eigenvalues for $\epsilon<\epsilon_{\text {bif }}$ (see Fig. 11). This means that $\epsilon_{\text {bif }}$ coalesces with $\epsilon_{\mathrm{c}}$ :

$$
\epsilon_{\mathrm{bif}}=\epsilon_{\mathrm{c}} \text {. }
$$

Although the instability mechanisms consists of one pair of complex conjugate eigenvalues after another crossing the imaginary axis, at $N \rightarrow \infty$ an infinite number of such pairs coalesce and an infinite number of oscillatory instabilities is unleashed within an arbitrarily small change in $\epsilon$.

For $v \rightarrow 0$ the destabilization scenario of the fixed point is thus different than has been suggested in the light of "finite-size simulations" [5,9]. On one side of the phase transition we have totally stable behavior, while on the other side we have immediately an infinite number of unstable modes on all scales. These instabilities are purely exponential and not oscillatory. 


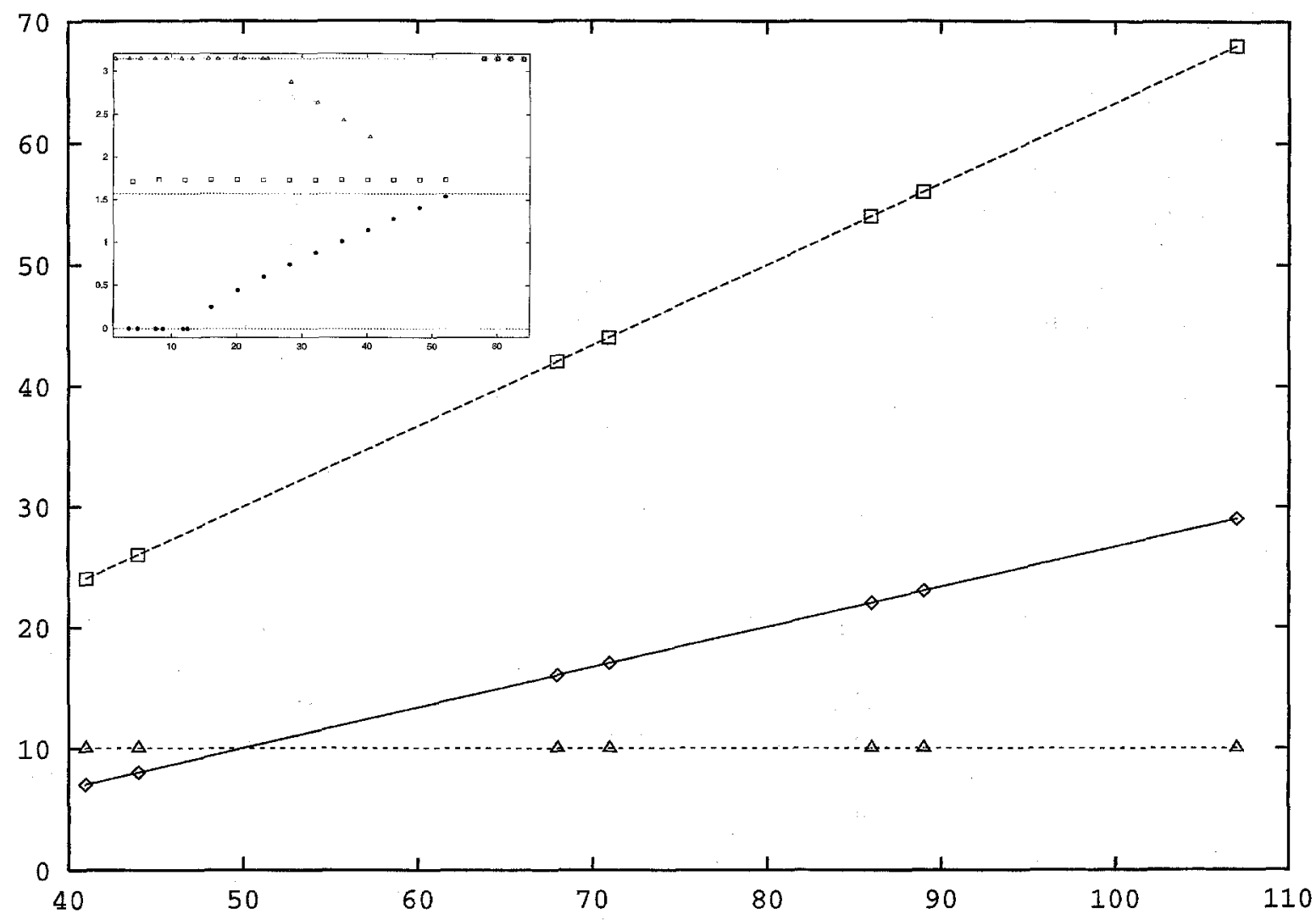

Fig. 9. Number of real (solid line), "straight" complex (dashed), and deviating eigenvalues (dots) with increasing shell number $N_{D}$. The slope of the lines is exactly $\frac{2}{3}, \frac{1}{3}$ and 0 , respectively. Inset: Main branch of "deviating" eigenvalues and real eigenvalues in a $\log _{\lambda}|\sigma|-\arg (\sigma)$ plot, for $\epsilon=0.33$ (triangles), $\epsilon=0.37$ (squares), and $\epsilon=0.4$ (circles). The tilted branch rotates with changing $\epsilon$ and is horizontal at the phase transition and unstable above (black). It can be seen that in the $N \rightarrow \infty$ limit an infinite number of eigenvalues turns unstable immediately above the phase transition.

\subsection{Asymptotics}

The phase transition is a change in the behavior of a very large number of eigenfunctions all at once. Biferale [11] has explained this phase transition as a blockage in the energy flow caused by a flow of helicity. Since the blockage can occur anywhere in the ISR it seems reasonable to assume that, when the conditions are right, blockages can occur at many places affecting many eigenfunctions and eigenvalues at once.

Another way of asking the same question is more mathematical in character. To see this phase transition we must have some kind of change in the small- $\nu$ asymptotics of the system. That is we must have some sort of change which can be seen by an eigenfunction with $j$ much larger than unity and much smaller than $N_{\mathrm{D}}$. So we need an asymptotic theory of eigenfunctions for this system.

To understand the phase transition, we must first understand how the phase sector can be so unstable. The key is given in Table 1. In that table we see that in contrast to the magnitude sector, the phase sector shows far more flexibility in the large- $n$ limit of the eigenfunction. The magnitude sector has but one value of $p_{1}$ and $p_{2}$ for all eigenfunctions in this region. The phase sector can actually have two different linear combinations. The two 

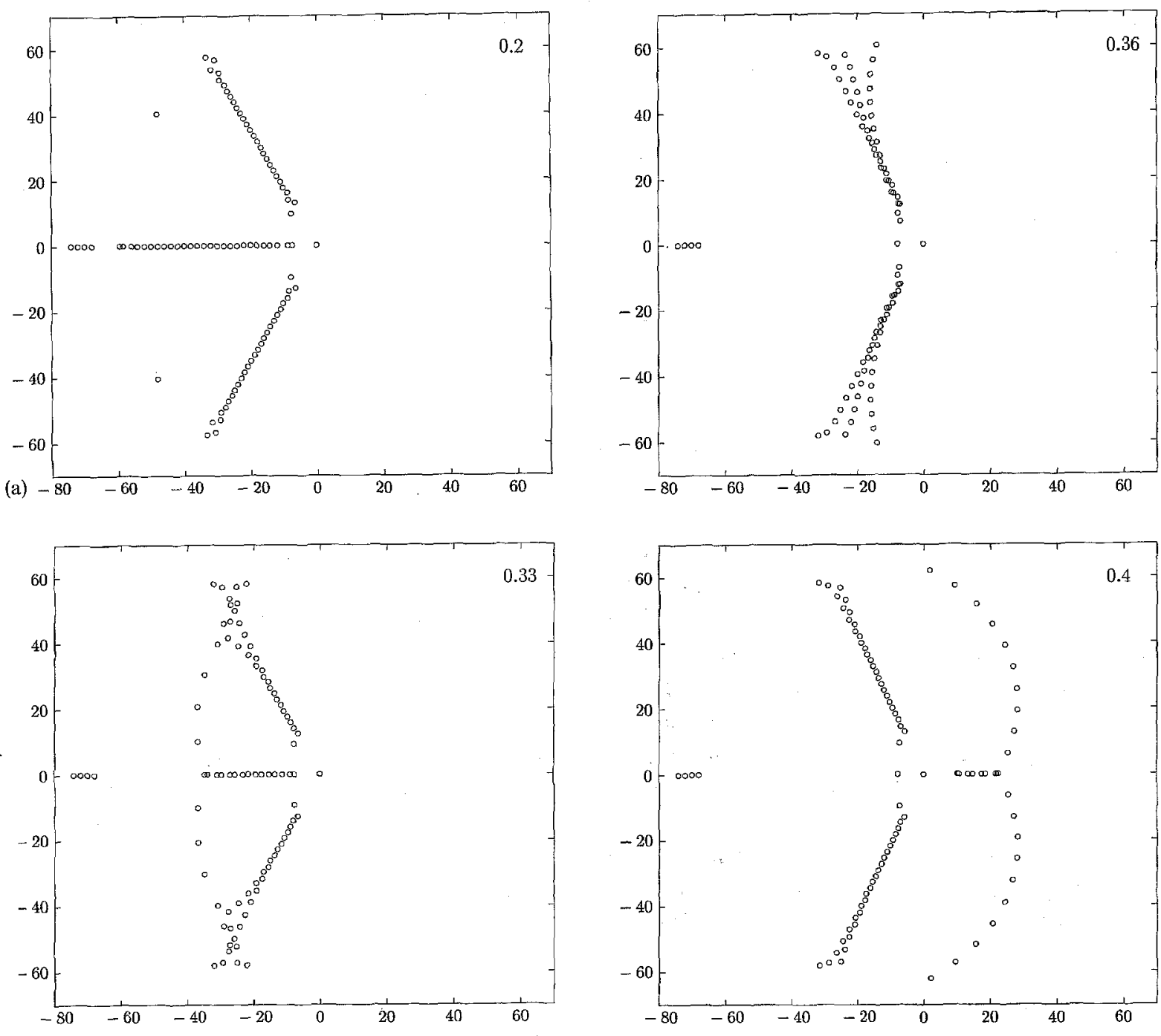

Fig. 10. This sequence of pictures shows the phase eigenvalues with changing $\epsilon$. We start with three completely stable branches in (a). Real eigenvalues turn complex and move towards the imaginary axis (b) until fewer and fewer real eigenvalues are left (c). Notice, all the "deviating" eigenvalues that do not lie on a straight line are finite size effects, i.e., they constitute only a negligible part for a sufficiently large matrix. These deviating eigenvalues form a straight line at $\epsilon_{\text {bif }}=0.37$.(Fig. 3). Now the spectrum consists of six complex branches and the scaling law (S) is no longer valid and has to be replaced by scaling law $\left(S^{2}\right)$. Above $\epsilon_{\text {bif }}(\mathrm{d})$ eigenvalues return to the real axis, but are now unstable. Essentially there are two complex branches and one real branch again as it was below $\epsilon_{\text {bif. }}$. An even higher value of $\epsilon=0.5$ is shown in Fig. 2.

permitted combinations are:

$$
\delta \Phi_{3 k+1}=0, \quad \delta \Phi_{3 k+2}=-\delta \Phi_{3 k}
$$

and also, for example,

$$
-\frac{1}{2} \delta \Phi_{3 k+1}=\delta \Phi_{3 k+2}=\delta \Phi_{3 k}
$$




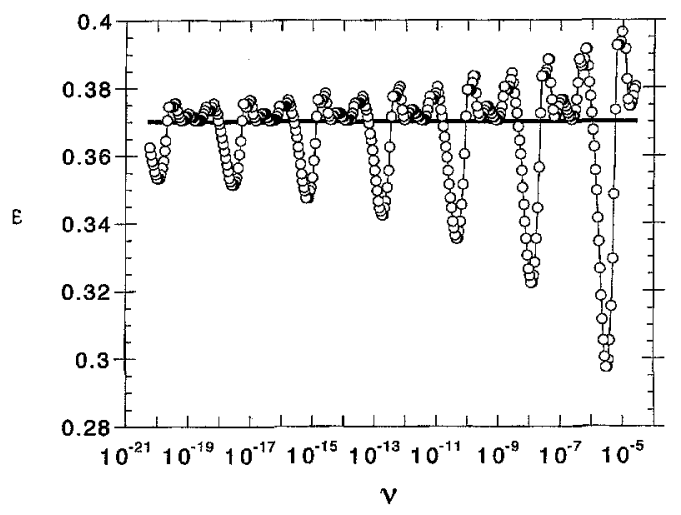

Fig. 11. Border of stability $\epsilon_{\mathrm{c}}$ as a function of $\nu$ for $\lambda=2 . \epsilon_{\mathrm{c}}$ approaches the Biferale prediction (thick horizontal line) for small $\nu$. Some of the points are due to a complex pair of eigenvalues passing through the imaginary axis, others are due to a single real eigenvalue going through zero.

for integer values of $k$. The changes in expression (38) are an exact symmetry of the GOY system and produce a phase sector eigenfunction which has eigenvalue zero. The changes in expression (39) satisfy the eigenvalue equation for all $n$ values except $n=1$. Here, the eigenvalue equation fails because of the forcing term. However, this combination also forms a possible high- $n$ behavior. All eigenfunctions have one or another linear combination of these two behaviors as the high- $n$ limit. But there are two such linear combinations possible. In comparison to the magnitude case, we seem to have lost a boundary condition at the high- $n$ end.

Thus, we state the first contrast between the magnitude sector and the phase sector. The magnitude sector can be described by giving two boundary conditions at the high- $n$ side of the ISR; the phase sector can be described by giving only one.

We must have, someplace, an extra boundary condition for the phase sector. Bifarale directed our attention to the conservation of helicity. Instead of doing a full analysis of the possible extra conditions, we just follow his direction and look at the helicity conservation law, Eq. (36b), in the phase sector. (The reader will recognize that some leap of faith is required around this point in the argument.) This equation is

$$
\sum_{n} h^{n}\left(u_{n}\right)^{2} \delta \Phi_{n}^{j}=-\frac{f h u_{1} \delta \Phi_{1}^{j}}{\sigma_{\phi}^{j}} .
$$

But, for an eigenvalue with $j$ in the middle of the ISR, the first component of the eigenfunction is very, very small. This component goes to zero more rapidly than an exponential in $j$. For this reason, it is quite reasonable to neglect the right-hand side of the helicity identity and write instead:

$$
\sum_{n} h^{n}\left(u_{n}\right)^{2} \delta \Phi_{n}^{j}=0 \text { for } j \text { in region } \mathrm{I} .
$$

We propose that this identity replaces the lost high- $n$ boundary condition for the asymptotics of the phase sector.

This proposition has several quite attractive features. We expect the eigenfunction to be of roughly the same order of magnitude over the whole range in which $n$ is greater than $j$. Then for $\epsilon<\epsilon_{\text {bif }}$ the summation has its main contribution at the lower- $n$ end and then falls off as

$$
\delta \Phi_{n}^{j}\left(-\frac{1-\epsilon_{\mathrm{bif}}}{1-\epsilon}\right)^{n}
$$


When we are at the critical point, the summation does not fall at all but the summand looks like

$$
\delta \Phi_{n}^{j}(-1)^{n} .
$$

Finally, above the critical point the major contributions come at the high- $n$ end and then fall off toward lower $n$ with the same law as shown in Eq. (42a). Near the phase transition, these behaviors produce long-range correlations between the different parts of the ISR. It is these correlations which form the key to the phase transition.

From these assumptions, one can find scaling laws for the phase eigenvalues, namely on the small- $j$ end

$$
\sigma^{\prime j}=\sigma^{j}\left(1+\mathrm{O}\left(\frac{1-\epsilon_{\mathrm{bif}}}{1-\epsilon}\right)^{N_{\mathrm{D}}-j}\right) \quad \text { for } j \text { in region } \mathrm{S}
$$

Here, as before, the prime indicates a decrease in the viscosity by a factor of $\lambda^{4}$ together with a shift in the cutoff, $N$. The corresponding result on the large- $j$ end is the statement, which we have used before.

$$
\sigma^{\prime(j+3)}=\lambda^{2} \sigma^{(j)}\left(1+\mathrm{O}(\epsilon-1)^{j}\right) \text { for } j \text { in region } \mathrm{L} \text {. }
$$

Both together give the scaling law:

$$
\sigma^{(j+3)}=\lambda^{2} \sigma^{(j)} \quad \text { for } j \text { in region } \mathrm{I} \text {. }
$$

To check the accuracy of our thinking we show in Fig. 7 polar plots in which we overlay the eigenvalues $\sigma_{j}$ with, respectively, the eigenvalues $\sigma_{j}^{\prime}$ and $\lambda^{-2} \sigma_{j+3}^{\prime}$. According to the theory, the first of these should agree very well for small magnitudes of the eigenvalue, while the second should agree on the large magnitude end. The figures bear this out. For a more accurate check, we construct errors as, for example,

$$
\delta=\left|\sigma_{j} / \sigma_{j}^{\prime}\right|-1
$$

These errors are shown in Fig. 8 and compared with the theoretical estimates taken from Eqs. (43a) and (43b). Since the estimated and the actual errors agree rather well, we can argue that we have the essence of the phase eigenvalues.

\section{Summary and conclusions}

The GOY model has a linear response behavior about a static solution in which the stability eigenvalues extend over a huge range of frequencies. In the ISR, the eigenvalues show a scaling behavior limited by disturbances from stirring or from viscous effects. The scaling of the eigenvalues is more complex in the phase sector than in the magnitude response. The phase sector shows a phase transition connected with a boundary condition which moves from one end of the ISR to the other. This boundary condition is derived from the conservation law for the model's version of helicity. Eigenvalues change quite abruptly as the model's parameter passes through its phase-transition value.

Much of the scaling mirrors the scaling properties of the static GOY model. However, there is scaling associated with the phase transition which we have not investigated in any detail. We have also not fully established the scaling behavior of the linear-stability eigenvectors. These studies are left for the future.

For now, we have demonstrated scaling in the linear response of a still system. We have seen a new phase transition and gained a qualitative understanding of its source. 


\section{Acknowledgements}

It is our pleasure to thank Luca Biferale, Jean-Philippe Brunet, Peter Constantin, Greg Huber, and Norman Lebovitz for helpful discussion. We are also indebted to Peko Hosoi, who programmed our IDL animations. This work has been supported by the ONR, the DOE contract number DE-FG02-92ER25119, the MRSEC Program of the National Science Foundation under Award Number DMR-9400379, and by the Deutsche Forschungsgemeinschaft (DFG) through its SFB185.

\section{Appendix A. Large scale forcing}

Most studies of the GOY model employed $F_{n}=f \delta_{n, 4}$ as large scale forcing. Though this forcing seems innocent, it has some disadvantages. The velocity component $u_{3}$ is much smaller than $u_{1,2,4,5,6, \ldots}$ [9], which is clearly unphysical. We force the system on level $n=1$. As can be seen from Eqs. (2) and (5), with $u_{3}=0$ and $n \rightarrow n-3$, the shift in the forcing by three shells just shifts the solution by three shells as well. ${ }^{5}$

With the different forcing the borderline of stability becomes only slightly different. For instance, $\epsilon_{\mathrm{c}}$ beyond which (2) becomes unstable is in the standard case $\left(\lambda=2, v=10^{-7}, f=\sqrt{2} \times 5 \times 10^{-3}\right.$ ) shifted from 0.370 to 0.377. More significant is the change in the amplitude of the $\epsilon_{\mathrm{c}}$ oscillations with viscosity. This viscosity dependence is shown in Fig. 11 for $\lambda=2$, and looks similar for other values of $\lambda$. In contrast to the case with forcing on the fourth shell (see [9], Fig. 12) the stability border $\epsilon_{\mathrm{c}}$ now approaches the constant $\epsilon_{\text {bif }}$ for vanishing viscosity $v \rightarrow 0$,

$$
\lim _{\nu \rightarrow 0} \epsilon_{\mathrm{c}}=\epsilon_{\mathrm{bif}}:=1-\lambda^{-2 / 3}
$$

Some of the transitions to instability in Fig. 11 are due to a complex pair of eigenvalues passing through the imaginary axis, others are due to a single real eigenvalue going through zero. ${ }^{6}$

In the main text we have studied the behavior of the overall eigenvalue spectrum. The stability border, on the other hand, is determined only by the first eigenvalue crossing the imaginary axis. Nevertheless, in the main text we have two strong evidences for a dependence of the stability border on the particular forcing. As we have seen in Section 3.2 the modulus of the eigenvalues is set by the cascade term. The eigenvalues that decide the stability are the small ones close to zero. Their size should depend on how the solution looks like at the very first shells, and this is obviously influenced by the kind of forcing. Also formula (36b) shows that the stability border should depend on the particular forcing.

\footnotetext{
${ }^{5}$ With the forcing $F_{n} \propto \delta_{n, 1}$ a static solution of Eq. (2) exists for all $0<\epsilon<1$, i.e., the saddle node bifurcation found in [9] is an artifact of the forcing $F_{n} \propto \delta_{n, 4}$. In particular, the overall existence of the static solution now allows for an analysis of the eigenvalue spectrum for the parameter values $\lambda=2$ and $\epsilon=0.5$ [2-4]

The artificially small $u_{3}$ (for $F_{n} \propto \delta_{n, 4}$-forcing) also leads to strongly non-normal eigenvectors. This was first discovered by Brunet [10], who also calculated the corresponding pseudoresonance. The reason for that can be seen from $(19 \mathrm{~b})$ and $(20 \mathrm{~b}):\left(A_{\phi}\right)_{3, m}$ with $m=1,2,4,5$ is small as $u_{3} \sim v$ is so small. All the eigenvectors of $A_{\phi}$ then have a huge 3rd component and are thus nearly parallel to each other. This makes $A_{\phi}$ very non-normal, the more, the smaller $v$ is.

${ }^{6}$ The model with the forcing on the fourth shell shows a transition from stable to unstable behavior only via two complex conjugated eigenvalues going through the imaginary axis. With the forcing on the first shell instability for some $v$ occurs via a real eigenvalue going through zero, followed by a series of Hopf bifurcations (take e.g. $v=10^{-8}, f=\sqrt{2} \times 0.005, k_{0}=0.0625$ ). However, from the viewpoint of the scenario of instability in the limit $v \rightarrow 0$, treated in Section 4.1, this additional type of transition is not fundamentally different from the other one.
} 


\section{Appendix B. A related phase transition}

In this appendix we analyze the related phase transition in the spectrum of the matrix

$$
A_{\xi}=\xi \cdot C-D
$$

The matrices $C$ and $D$ contain the solution of (2). For general $\xi$ the matrix $A_{\xi}$ is not the stability matrix of the system. Only for $\xi=-1$ we have $A_{-1}=A_{\mathrm{M}}$ and for $\xi=1$ we have $A_{1}=A_{\phi}$. The generalized amplitude matrix $A_{\xi}$ with $\xi<0$ shows the same features as $A_{M}$ itself. So we only study the interesting case of $A_{\xi}$ with $\xi>0$ which displays a phase transition at $\xi=1$. The advantage of studying this phase transition rather than the one in $\epsilon$ discussed in the body of the paper is that here the critical point $\xi=1$ does not depend on the viscosity. Thus finite size effects are less complicated than for the transition in the spectrum of $A_{\phi}$ at the $v$ dependent $\epsilon_{\mathrm{c}}$. Taking $\xi \approx 1$ (but $\xi \neq 1$ ) can be thought of as equivalent to slightly varying the dynamical equation (2) and thus changing the phase symmetry of that equation, which leads to a different solution. We perform the analysis for $\epsilon=0.3$ as it is the spurious stabilization of the phase matrix for $\epsilon<\epsilon_{\mathrm{c}} \approx 0.37$ which we want to understand.

We start with $\xi=0$. All eigenvalues $-v k_{n}^{2}$ of $A_{0}=-D$ are of course stable. However, with increasing $\xi$, more and more eigenvalues of $A_{\xi}$ turn positive as soon as $\xi \gtrsim \nu$. This feature holds for $|\lg \nu| \sim 30$ orders of magnitude in $\xi$. From comparing the viscous and the inertial contribution to $A_{\xi}$ we obtain that the largest eigenvalue increases as

$$
\sigma_{\max } \sim \xi^{3 / 2} v^{-1 / 2}
$$

$\sigma_{\max }$ becomes as large as $10^{12}$ for $\xi \approx 0.63$ and the standard parameters $\lambda=2, \epsilon=0.3, N=81, v \sim 10^{-30}$. This is the behavior we see in the spectrum of $A_{\phi}=A_{1}$ for $\epsilon>\epsilon_{\mathrm{c}}$ and what we had expected also for smaller $\epsilon$.

Why and how is stability achieved for $\xi=1$, i.e., why does $A_{1}=A_{\phi}$ not have any positive eigenvalue for $\epsilon<\epsilon_{\mathrm{c}}$ ?

For $\xi$ growing beyond 0.63 towards 1, two real eigenvalues merge, form a complex pair which moves in the complex plane on a circle and finally turns stable through an inverse Hopf bifurcation. This happens again and again through a self-similar cascade of bifurcations towards phase symmetry at $\xi=1$ - no unstable eigenvalue is left. This phase symmetry of $A_{1}=A_{\phi}$, discovered in [9], reflects itself in a zero eigenvalue, as discussed above.

We now analyze the singularity in detail. We introduce the distance $\tau$ from the singularity at $\xi=1$,

$$
\xi=1+\tau
$$

and increase $|\tau|$ on a $\log$ scale from $\tau=0$. In this way we break the phase symmetry in a stronger and stronger way.

We describe the features for $\tau \leq 0$. For $\tau=0$ we only have negative eigenvalues and one eigenvalue equals zero. This center manifold eigenvalue turns positive for $|\tau|>0$, signaling instability. Moreover, two different small (modulus wise) real eigenvalues $(<0)$ merge on the real axis and form a complex pair which wanders towards the $\mathbb{R} \sigma=0$ axis and turns unstable via a Hopf bifurcation. This happens as early as for $\tau \approx 10^{-10}$, see Fig. 12 . In the right complex half plane they continue to wander on a kind of circle, finally merging again on the real axis, now forming two positive real eigenvalues. But meanwhile a second pair of complex eigenvalues has formed in the left half plane which again turns unstable via a Hopf bifurcation and then finally merges. This happens again and again, leading to $3,5,7,9,11, \ldots$ positive eigenvalues. The real parts of the positive eigenvalues are plotted in Fig. 12. The self-similarity of the cascade of bifurcations can clearly be observed. The maximal eigenvalue $\sigma_{\max } \propto \tau^{4 / 3}$. The scaling law mirrors the classical K41 scaling of $u_{n}$.

For $\tau>0$ the behavior is very similar to the $\tau<0$ case. The only difference is that the center manifold (zero) eigenvalue turns stable first and we now have an even number $2,4,6,8, \ldots$ of positive eigenvalues. 


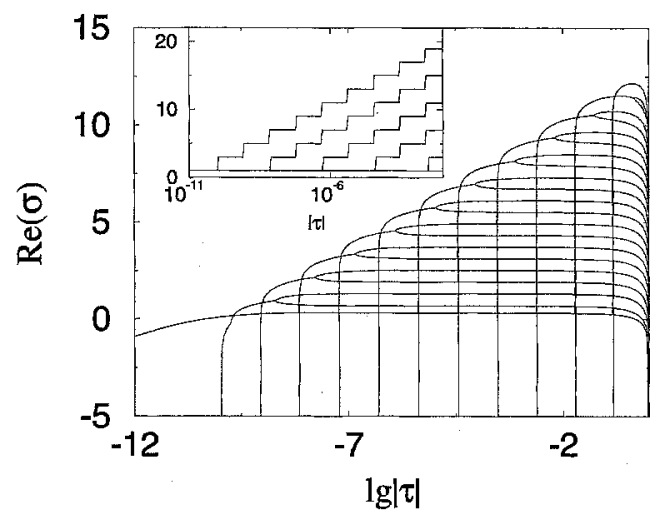

Fig. 12. All positive eigenvalues of $A_{\xi}$ for $\tau=\xi-1$ between 0 and -1 on a $\log -\log$ scale in $|\tau|$, corresponding to a $\lg$ versus $\lg \lg \xi$ plot. The self-similar cascade of bifurcations towards phase symmetry has its origin in the self-similarity of the matrix itself. The parameters are $N=80, \nu \sim 2 \times 10^{-29}, \epsilon=0.3, \lambda=2$. For $\tau=-1$ (right edge of the plot) we have $A_{\xi}=-D$ and all eigenvalues are again stable. The inset shows the number of positive eigenvalues of $A_{1-\tau}$ as a function of $|\tau|$ for various system sizes $N=80,68,56,44,32,20$, left to right, corresponding to viscosities $v=v_{0} \times 16^{i}, i=0,4,8,12,16,20$, respectively. $v_{0} \sim 2 \times 10^{-29}, \epsilon=0.3$.

To demonstrate the finite size effects of the transition we plot the number of positive eigenvalues as a function of $\lg \tau$ for various system sizes $N$, i.e., viscosities $\nu$, see the inset of Fig. 12. In the $N \rightarrow \infty$ limit, even for an arbitrarily small $|\tau|$, we have an infinite number of unstable eigenvalues.

\section{References}

[1] E.B. Gledzer, Sov. Phys. Dokl. 18 (1973) 216.

[2] M. Yamada and K. Ohkitani, J. Phys. Soc. Jpn. 56 (1987) 4210; Prog. Theor. Phys. 79 (1988) 1265; K. Ohkitani and M. Yamada, Prog. Theor. Phys. 81 (1989) 329.

[3] M.H. Jensen, G. Paladin and A. Vulpiani, Phys. Rev. A 43 (1991) 798; D. Pisarenko et al., Phys. Fluids A 5 (1993) 2533.

[4] R. Benzi, L. Biferale and G. Parisi, Physica D 65 (1993) 163.

[5] L. Biferale, A. Lambert, R. Lima and G. Paladin, Physica D 80 (1995) 105.

[6] L. Kadanoff, D. Lohse, J. Wang and R. Benzi, Phys. Fluids 7 (1995) 617.

[7] L. Kadanoff, Physics Today 48 (1995) 11.

[8] R. Benzi, L. Biferale, R. Kerr and E. Trovatore, Phys. Rev. E, (1996), in press.

[9] N. Schörghofer, L. Kadanoff and D. Lohse, Physica D 88 (1995) 40.

[10] J.P. Brunet, private communication (1995).

[11] L. Biferale, private communication (1995).

[12] L. Biferale and R. Kerr, Phys. Rev. E 52 (1995) 6113. 